\title{
Modelo experimental de hepatectomia parcial laparoscópica em ratos $^{1}$
}

\author{
Fabrício Mascarenhas de Oliveira ${ }^{2}$ \\ Lúcio Couto de Oliveira $\mathrm{Jr}^{3}$ \\ Vanessa Alves Costa ${ }^{4}$ \\ Mario Castro Carreiro \\ Pedro Guimarães ${ }^{6}$
}

\begin{abstract}
Oliveira FM, Oliveira Jr LC, Costa VA, Carreiro MC, Guimarães. Modelo experimental de hepatectomia parcial laparoscópica em ratos. Acta Cir Bras [serial online] 2003 Maio-Jun;18(3). Disponível em URL: http://www.scielo.br/acb.
\end{abstract}

RESUMO - Objetivo: Descrever um modelo experimental de hepatectomia parcial laparoscópica em ratos. Métodos: Foram utilizados 35 ratos Rattus Norvegicus albinus da linhagem Wistar todos machos pesando $250+/-50 \mathrm{~g}$. Os animais foram anestesiados com cetamina e xilasina, e submetidos à insuflação através da agulha de Veress com $\mathrm{PCO} 2 \mathrm{de} 7 \mathrm{mmHg}$. Após o pneumoperitôneo foram transpassados pela parede abdominal dois trocateres com 5 e um com $11 \mathrm{~mm}$ de espessura, de tal modo a formarem uma figura geométrica triangular. A ligadura de três lobos hepáticos (esquerdo, central esquerdo e central direito) foi feita utilizando-se o "endoloop" e a eletrocoagulação dos pontos hemorrágicos através do "hook". Os animais foram sacrificados após um período de observação de oito dias. Resultados: Três animais $(8,6 \%)$ morreram na indução anestésica. Após um período de observação de oito dias 30 animais sobreviveram $(85,7 \%)$, e apenas $2(5,7 \%)$ vieram a óbito no pós-operatório imediato. Quanto às complicações, a presença de granuloma foi observada em $54,3 \%$ dos ratos $(\mathrm{n}=19)$. Nenhuma conversão para a cirurgia aberta foi necessária. Conclusão: O modelo de hepatectomia parcial laparoscópica em ratos utilizando material cirúrgico semelhante ao usado em humanos é factível para o treinamento e aprimoramento de novos cirurgiões.

DESCRITORES - Cirurgia laparoscópica. Hepatectomia parcial. Ratos.

\section{Introdução}

A primeira técnica de hepatectomia parcial convencional em ratos, onde há descrição de exérese de $70 \%$ do peso do fígado com sucesso, foi publicada em $1931^{(1)}$. Até os dias atuais, a mesma técnica é amplamente utilizada como base para os estudos desta natureza.
A cirurgia laparoscópica mudou as diretrizes da abordagem abdominal, o que vem se refletindo de positivamente na cirurgia experimental, na medida em que novos métodos e técnicas vêm sendo desenvolvidos e aprimorados. Os estudos pioneiros utilizavam porcos e cães, pois a cavidade abdominal destes animais possui um tamanho compatível com a dos humanos. Mais recentemente a cirurgia minimamente invasiva vem

1. Trabalho realizado na Escola Bahiana de Medicina e Saúde Pública - EBMSP-BA.

2. Acadêmico do $5^{\circ}$ Ano de Medicina da Escola Bahiana de Medicina e Saúde Pública (EBMSP-BA).

3. Acadêmico do $6^{\circ}$ Ano de Medicina da Escola Bahiana de Medicina e Saúde Pública (EBMSP-BA).

4. Acadêmica do $4^{\circ}$ Ano de Medicina da Escola Bahiana de Medicina e Saúde Pública (EBMSP-BA).

5. Professor Adjunto IV da Universidade Federal da Bahia (UFBA). Coordenador da Disciplina de Técnica Operatória e Cirurgia Experimental da Universidade Federal da Bahia (UFBA).

6. Professor Adjunto da Disciplina de Técnica Operatória e Cirurgia Experimental da Universidade Federal da Bahia (UFBA). Doutor pela Escola Paulista de Medicina da Universidade Federal de São Paulo (EPM-UNIFESP). 
sendo aprimorada, principalmente no que diz respeito ao material de menor calibre, e desta maneira iniciou-se a utilização de coelhos e ratos em alguns trabalhos ${ }^{(2,3,4)}$.

Todos os estudos encontrados na literatura mundial descrevem modelos de cirurgia laparoscópica com material adaptado para ratos ${ }^{(23,4)}$. Apenas um descreve a hepatectomia por via laparoscópica ${ }^{(2)}$. Na literatura nacional, não foi encontrado nenhum artigo referente à utilização da laparoscopia em ratos.

O objetivo desta comunicação é descrever um modelo de hepatectomia parcial laparoscópica em ratos.

\section{Métodos}

Os experimentos foram aprovados pela Comissão de Ética da Escola Bahiana de Medicina e Saúde Pública (E.B.M.S.P. -BA).

Para o presente estudo foram utilizados 35 ratos Rattus norvegicus albinus da linhagem Wistar, procedentes do biotério da E.B.M.S.P. - BA, pesando $250+/-50 \mathrm{~g}$, com idade média de 3 meses sendo todos machos. Os animais foram colocados em gaiolas individuais, medindo $20 \mathrm{X} 30 \mathrm{X} 40 \mathrm{~cm}$, aclimatados durante 15 dias a uma temperatura de $25^{\circ} \mathrm{C}$, em períodos de 12 horas luz e 12 horas escuridão com ração e água livres. Todos os animais foram avaliados no intra-operatório quanto a presença ou não de hemorragia controlável, e após um período de 8 dias quanto a presença ou ausência de: áreas de hemorragia intra-abdominal, granuloma, abscesso, deiscência de sutura e necrose.

\section{Pré-operatório}

Cerca de oito horas antes do procedimento os animais foram colocados em jejum, e anestesiados por via intra-peritoneal com cetamina $(7 \%)$ e xilasina $(0.3 \%)$ na proporção de $2: 1 \mathrm{ml}$, com $0,2 \mathrm{ml} / 100 \mathrm{~g}$ de peso da solução. Em seguida, foram posicionados na mesa cirúrgica em decúbito dorsal, tricotomizados e submetidos à antissepsia.

\section{Ato operatório}

Após a colocação dos campos, foi feita uma incisão com $0.5 \mathrm{~cm}$ de comprimento com lâmina de bisturi frio número 15 , a uma distância de $2 \mathrm{~cm}$ abaixo do rebordo costal esquerdo. Realizou-se a fixação da musculatura abdominal com as pinças de Alis, para tracionamento da parede abdominal, com o intuito de facilitar a passagem da agulha de Veress a ângulo de $90^{\circ}$ através da mesma.

Foram feitos testes de segurança, conexão da mangueira do insuflador e início do pneumoperitôneo com PCO2 de $7 \mathrm{mmHg}$. A segunda incisão com 1.0 $\mathrm{cm}$ de comprimento foi realizada distando $0.5 \mathrm{~cm}$ acima da sínfise pubiana, através da qual atingiu-se "às cegas" a cavidade abdominal com trocater de $11 \mathrm{~mm}$, o qual foi fixado com sutura em bolsa para impedir a perda de $\mathrm{CO} 2$ durante o procedimento.

A óptica de $10 \mathrm{~mm}$ foi introduzida e, através da sua imagem ou "visão direta", a agulha de Veress foi retirada e um trocater com $5 \mathrm{~mm}$ introduzido em seu lugar. Então, uma terceira e última incisão foi feita distando $2.0 \mathrm{~cm}$ abaixo do rebordo costal direito e medindo $0.5 \mathrm{~cm}$, através da qual, sob visão direta, um terceiro trocater com $5 \mathrm{~mm}$ de diâmetro foi posicionado (Figura 1).

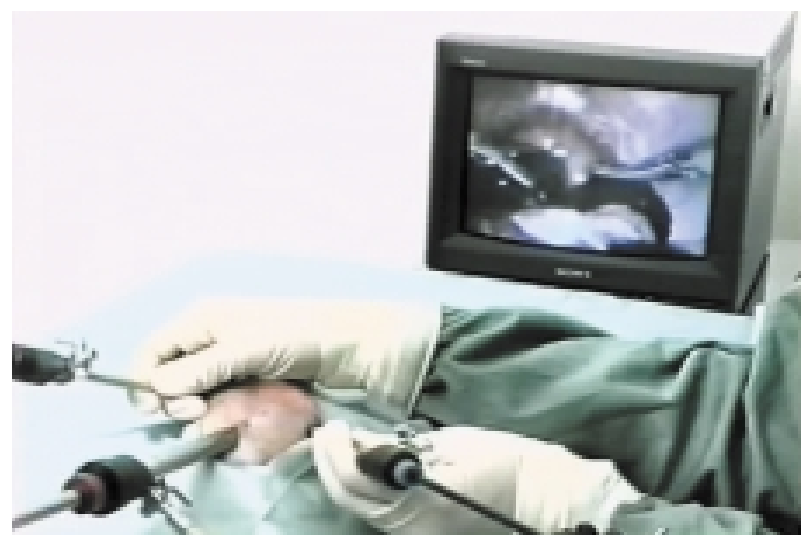

FIGURA 1 - Imagem externa do procedimento mostrando o posicionamento dos trocaters na cavidade abdominal do rato.

Já na cavidade, localizou-se o fígado e, com uma pinça, o seu lobo esquerdo foi tracionado. O "endoloop" (nó de forca com fio categute 3.0) foi introduzido para o interior da cavidade abdominal via trocater, e através deste, o cirurgião passou o conjunto (pinça atraumática - lobo hepático) e tracionou o fio (Figura 2).

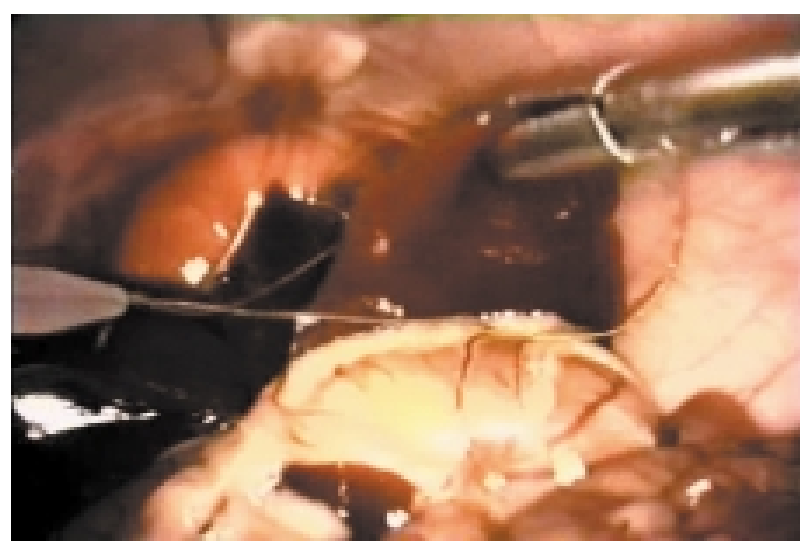

FIGURA 2 - Imagem intra-abdominal a partir do laparoscópio mostrando a passagem do Endoloop através do lobo esquerdo do fígado com auxílio da pinça.

Esse tracionamento diminuiu o diâmetro da forca e consequientemente ocorreu a ligadura do pedículo vascular e do parênquima hepático. O fio catgut 3-0 
foi exposto para o corte, o tubo do endoloop com o seu redutor retirado e, em seu lugar, a pinça atraumática foi re-colocada permitindo que o cirurgião tracionasse o fígado, facilitando sua ressecção acima da ligadura, pela tesoura curva (Figura 3 ).

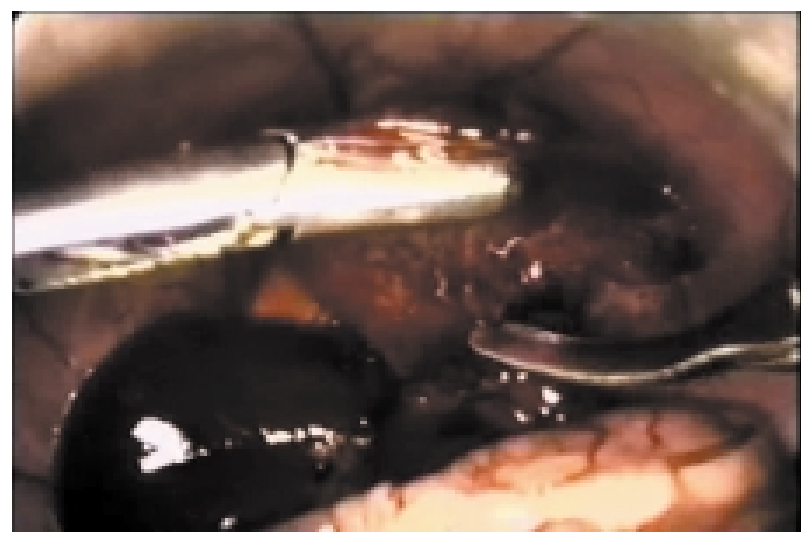

FIGURA 3 - Imagem intra-abdominal via laparoscópio, onde pode ser observada a ressecção do lobo esquerdo com a tesoura após a ligadura com o Endoloop.

O lobo central esquerdo foi posicionado no sentido caudal com o auxilio da pinça e novamente foi feita a ligadura deste lobo através do Endoloop 3-0, com corte do fio, ressecção do órgão e por fim cauterização dos pontos hemorrágicos. Por fim, localizou-se o lobo central direito, e procedeu-se de maneira semelhante,
Feito isto, o lobo esquerdo foi colocado sob as alças abdominais e o "hook" então iniciou o seu papel de eletrocoagulação dos pontos hemorrágicos, já que o Endoloop não foi suficiente para realizar a hemostasia por completo (Figura 4).

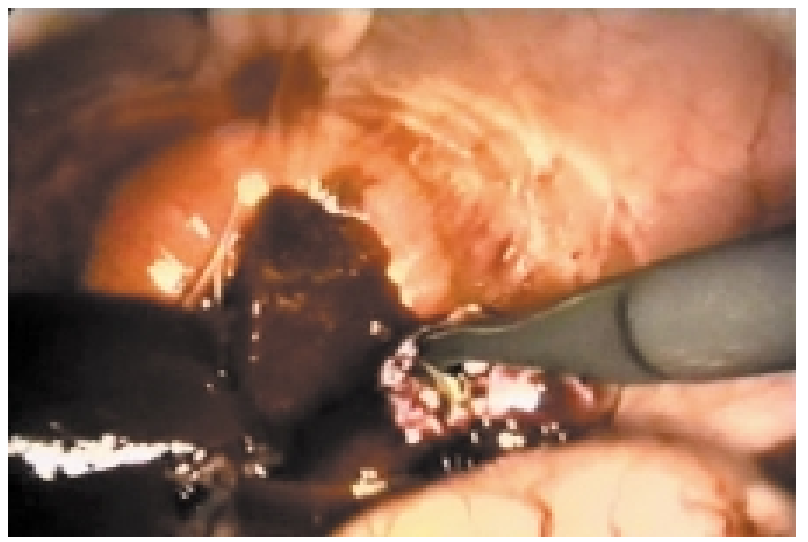

FIGURA 4 - Imagem intra-abdominal mostrando a cauterização dos pontos hemorrágicos através do "Hook" após a ressecção do lobo esquerdo.

ou seja, introdução do Endoloop e ligadura com posterior ressecção e cauterização (Figura 5A, B e C).

Para finalizar, realizou-se a extirpação dos três lobos hepáticos através da primeira incisão com movimentos rotatórios e delicados (Figura 5D).

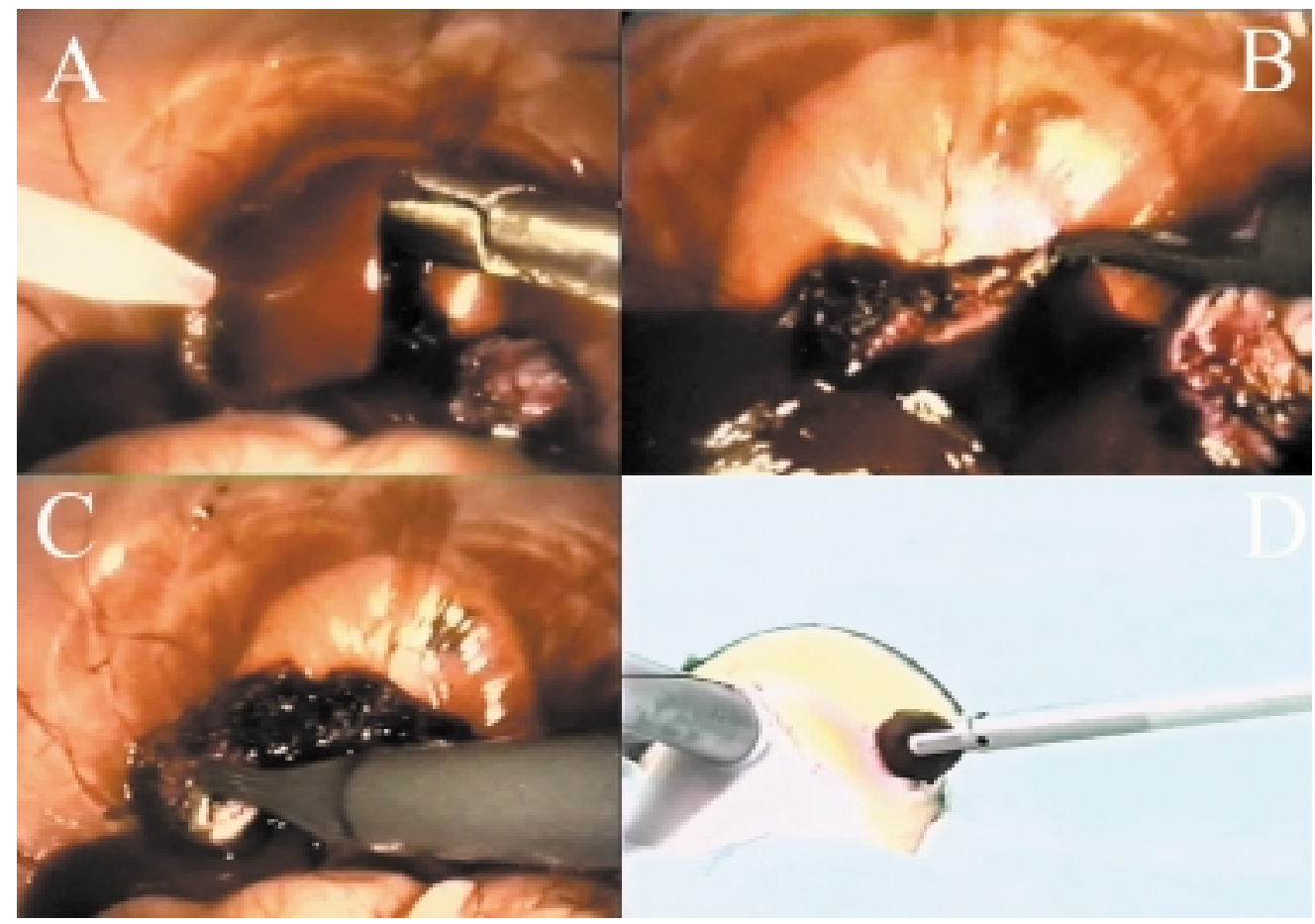

FIGURA 5 - A. Ligadura do parênquima hepático (lobo medial esquerdo) com endoloop; B. Cauterização dos pontos hemorrágicos do lobo medial esquerdo com hook; C. Cauterização dos pontos hemorrágicos do lobo medial direito com hook; D. Imagem externa mostrando a exérese dos lobos hepáticos via incisão com auxílio da pinça. 
Fez-se a inspeção final da cavidade, retiraram-se os trocateres sob visão direta, e a parede abdominal foi suturada em dois planos.

\section{Pós-operatório}

Os animais foram re-colocados em gaiolas individuais e, observados durante oito dias, nas mesmas condições já citadas (ver método). Após um período de observação de oito dias os animais foram sacrificados em uma câmara fechada com éter etílico a $50 \%$ e submetidos à autópsia.

\section{Resultados}

O tempo operatório médio foi de $30+/-10$ minutos. A mortalidade durante o procedimento anestésico foi observada em $8,6 \%$ dos animais $(n=3)$. A mortalidade no pós-operatório imediato foi constatada em $5,7 \%$ da amostra $(\mathrm{n}=2)$. A taxa de sobrevida após um período de observação de 8 dias foi de $85,7 \%(n=30)$. Áreas de hemorragia foram facilmente controladas com o uso do Endoloop e do eletrocautério, exceto em 5,7\% da amostra total $(\mathrm{n}=2)$. Analisando a taxa de hemorragia controlável apenas nos animais submetidos ao procedimento, notamos que o percentual aumentou para $6,3 \%(n=2 / 32)$.

$\mathrm{Na}$ autópsia observou-se a presença de granuloma ao redor da ligadura hepática com o fio categute 3.0 em 54,3\% ( $n=19)$ dos ratos. Em nenhum dos animais operados foi necessária a conversão para a cirurgia aberta. Não foram observados sinais de infecção intraabdominal, deiscência de sutura e nem áreas de necrose.

\section{Discussão}

$\mathrm{Na}$ literatura, encontramos alguns relatos sobre o uso da vídeo cirurgia experimental, principalmente em porcos e cães ${ }^{(5-7)}$. Ao nosso ver este tipo de modelo é de extrema importância para o treinamento e aperfeiçoamento de novos médicos que queiram aprimorar os conhecimentos cirúrgicos ${ }^{(5-8)}$. Entretanto, observamos que o uso de animais com porte menor como os ratos ainda têm uso mais restrito, apesar de existirem na literatura a descrições de alguns procedimentos laparoscópicos ${ }^{(2-7)}$.

Sabe-se que os modelos experimentais em ratos contribuem para o desenvolvimento da cirurgia, principalmente no aprimoramento de novas técnicas ${ }^{(2,3)}$. Por isto é de fundamental importância introduzir em nosso meio a vídeo cirurgia neste animal de baixo custo e fácil manuseio. A única técnica descrita de hepatectomia parcial laparoscópica utilizou material adaptado ou seja de menor calibre, o que obviamente gera custos maiores ${ }^{(2)}$. Em nosso estudo, utilizamos o mesmo material da cirurgia em humanos sem adaptação de nenhum instrumental, sem prejuízo quanto a taxa de morbi-mortalidade destes animais.

A morte pós-operatória ocorreu nos mesmos dois animais $(5,7 \%)$ com presença de hemorragia de difícil controle, o que aconteceu ao nosso ver devido à inexperiência inicial do cirurgião, já que se tratavam dos primeiros animais operados. A presença de granuloma foi observada em $54,3 \%$ dos animais $(n=19)$, concordando com os dados de literatura ${ }^{(2)}$, que relata a presença em $71 \%(n=10 / 14)$ e pode ser explicada pela resposta inflamatória ao fio. Esta menor incidência de formação do granuloma, entretanto, pode ser explicada pelo menor tempo de observação comparando-se o nosso estudo com a literatura ${ }^{(2)}$ e pela morte no pósoperatório imediato de dois ratos.

As dificuldades da cirurgia laparoscópica (visão bidimensional, imagem virtual, ausência de tato) são semelhantes. Entretanto, com o treinamento em simuladores e conhecimento do material, mesmo os iniciantes são capazes de realizar tal procedimento de maneira eficaz e precisa.

A inovação deste estudo refere-se à realização de uma trilobectomia hepática por laparoscopia, o que nunca fora antes descrita na literatura mundial. No que se refere à publicação nacional, este trabalho parece ser o pioneiro na utilização de ratos para a abordagem laparoscópica. Esperamos que possa contribuir para o desenvolvimento de novas pesquisas dentro da laparoscopia experimental.

\section{Conclusão}

O modelo de hepatectomia parcial laparoscópica em ratos, utilizando o mesmo material cirúrgico usado em humanos, é factível para o treinamento e o aprimoramento de novos cirurgiões.

\section{Referências}

1. Higgins,G.M., Anderson, R.M. Experimental pathology of the liver. I. Restoration of the liver of the white rat following partial surgical removal. Arch Pathol 1931;12:186-02.

2. Krahenbühl, L., Feodorovici, M., Renulli, P., Schafer, M., AbouShady, M., Bauer, H.V. Laparoscopic partial hepatectomy in the rat: a new resectional technique. Dig. Surg 1998;15:140-4.

3. Filmar,F., Gomel, V., McComb, PF. Operative laparoscopic versus open abdominal surgery: a comparative study on postoperative adhesion formation in the rat model. Fertil Steril 1987;3:486-9.

4. Berguer,R., Gutt.C.N. Laparoscopic colon surgery in a rat model: a preliminar report. Surg Endosc 1994;8:1195-7.

5. Kalach, A.C.; Baro, M.C. H.; Vorero, N.H.; Marcovich, D.L.;Rojas, A.C.; Barrera, R.C.;Chaba, S.S. La rata como 
modelo animal para la enseñanza e investigación de la cirugía laparoscópica. Cir General 1996;3: 221-7.

6. Gutt,C.N., Riemer, V., Brier, C., Berguer, R., Paolucci, V. Standardized technique of laparoscopic surgery in the rat. Dig Surg 1998;15:135-9.
7. Berguer,R., Gutt.C.N., Stiegmann GV. Laparoscopic surgery in the rat: description of a new technique. Surg Endosc 1993;7:345-47.

8. Giuffriga MC, Marquet RL, Kazemier G, Wittich P, Bouvy ND, Bruining HAS, Bonjer HJ. Laparoscopic splenectomy and nephrectomy in a rat model: description of a new technique. Sur Endosc 1997;11:491-4.

Oliveira FM, Oliveira Jr LC, Costa VA, Carreiro MC, Guimarães. An experimental model of laparoscopic partial hepatectomy in rats. Acta Cir Bras [serial online] 2003 May-Jun;18(3). Available from URL: http://www.scielo.br/acb.

ABSTRACT - Purpose: To describe a model of laparoscopic partial hepatectomy in rats. Methods: 35 male rats Rattus Norvegicus albinus Wistar weighing $250+/-50 \mathrm{~g}$ were used in this study. The animals were anaesthetised with ketamin and xilasin and insufflated with PCO2 of $7 \mathrm{mmHg}$ through a Veress needle. After the pneumoperitoneum, two $5 \mathrm{~mm}$ and a $11 \mathrm{~mm}$ trocars were passed through the abdominal wall, so as to form a triangular geometrical figure. The ligature of three hepatic lobes (left, median left and median right) was done with Endoloop and the electrocoagulation of hemorrhagic spots with the hook. The animals were sacrificed after an eight-day observation period. Results: Three animals $(8,6 \%)$ died at the anaesthetic induction. After an eight-day observation period, 30 animals survived $(85,7 \%)$ and only $2(5,7 \%)$ died immediately after the surgery. In relation to complications, granuloma was observed in $54,3 \%(\mathrm{n}=19)$. No conversion to open surgery was necessary. Conclusions: The model of laparoscopic partial hepatectomy in rats using surgical material similar to that used in humans is doable for surgeons' training and improvement.

KEY WORDS - Laparoscopic surgery. Partial hepatectomy. Rats.

Conflito de interesse: nenhum Fonte de financiamento: nenhuma

Correspondência:

Fabrício Mascarenhas de Oliveira

Av. Euclides da Cunha, 443/804

40150-120 Salvador - BA

fabriciomasc@uol.com.br.

Data do recebimento: 04/02/2003

Data da revisão: 15/02/2003

Data da aprovação: 01/03/2003 